\title{
Optical interferometry in the presence of large phase diffusion
}

\author{
Marco G. Genoni, ${ }^{1}$ Stefano Olivares, ${ }^{2,3,4}$ Davide Brivio, ${ }^{2}$ Simone Cialdi, ${ }^{2,5}$ Daniele Cipriani, ${ }^{2}$ Alberto Santamato, ${ }^{2}$ \\ Stefano Vezzoli, ${ }^{2}$ and Matteo G. A. Paris ${ }^{2,3, *}$ \\ ${ }^{1}$ QOLS, Blackett Laboratory, Imperial College London, London SW7 2BW, United Kingdom \\ ${ }^{2}$ Dipartimento di Fisica, Università degli Studi di Milano, I-20133 Milano, Italy \\ ${ }^{3}$ CNISM, UdR Milano Statale, I-20133 Milano, Italy \\ ${ }^{4}$ Dipartimento di Fisica, Università degli Studi di Trieste, I-34151 Trieste, Italy \\ ${ }^{5}$ INFN, Sezione di Milano, I-20133 Milano, Italy \\ (Received 18 December 2011; published 10 April 2012)
}

\begin{abstract}
Phase diffusion represents a crucial obstacle toward the implementation of high-precision interferometric measurements and phase-shift-based communication channels. Here we present a nearly optimal interferometric scheme based on homodyne detection and coherent signals for the detection of a phase shift in the presence of large phase diffusion. In our scheme the ultimate bound to interferometric sensitivity is achieved already for a small number of measurements, of the order of hundreds, without using nonclassical light.
\end{abstract}

DOI: 10.1103/PhysRevA.85.043817

PACS number(s): 42.25.Hz, 07.60.Ly, 42.87.Bg

\section{INTRODUCTION}

Optical interferometry represents a highly accurate measurement scheme with wide applications in many fields of science and technology [1-5]. Besides, the precise estimation of an optical phase shift is relevant for optical communication schemes where information is encoded in the phase of traveling pulses. Several experimental protocols have been proposed and demonstrated to estimate the value of the optical phase [6-11], thus showing the possibility to attain the so-called Heisenberg limit [12-22]. Recent developments also revealed the potential advantages of nonlinear interactions [23]. However, in realistic conditions, one has to retrieve phase information that has been unavoidably degraded by different sources of noise, which have to be taken into account in order to evaluate the interferometric precision [24]. The effects of imperfect photodetection in the measurement stage, or the presence of amplitude noise in the interferometric arms, have been extensively studied [25-35]. Only recently, the role of phasediffusive noise in interferometry has been theoretically investigated for the optical polarization qubit [36-38], condensate systems [39,40], Bose-Josephson junctions [41], and Gaussian states of light [42]. As a matter of fact, phase-diffusive noise is the most detrimental for interferometry, and any signal that is unaffected by phase-diffusion is also invariant under a phase shift and thus totally useless for phase estimation.

In this paper, we present an experimental interferometric scheme where phase diffusion may be inserted in a controlled way, and demonstrate that homodyne detection and coherent signals are nearly optimal for the detection of a phase shift in the presence of large phase diffusion. Indeed, while in ideal conditions a squeezed vacuum is the most sensitive Gaussian probe state for a given average photon number [43], for large phase-diffusive noise, coherent states become the optimal choice, outperforming squeezed states [42]. In our scheme the ultimate bound to interferometric sensitivity, as dictated by the Cramér-Rao (CR) theorem, is achieved already for a small number of repeated measurements, of the order

*matteo.paris@fisica.unimi.it of hundreds, using Bayesian inference on homodyne data and without the need of nonclassical light.

The paper is structured as follows: In Sec. II we describe the evolution of a light beam in a phase diffusing environment as well as the bound to interferometric precision in the presence of phase noise. In Sec. III we describe our experimental apparatus, whereas the experimental results are reported and discussed in Sec. IV. Section V closes the paper with some concluding remarks.

\section{INTERFEROMETRY IN THE PRESENCE OF PHASE DIFFUSION}

The evolution of a light beam in a phase diffusing environment is described by the master equation

$$
\dot{\varrho}=\Gamma \mathcal{L}\left[a^{\dagger} a\right] \varrho,
$$

where $\mathcal{L}[O] \varrho=2 O \varrho O^{\dagger}-O^{\dagger} O \varrho-\varrho O^{\dagger} O$ and $\Gamma$ is the phase damping rate. An initial state $\varrho_{0}$ evolves as

$$
\varrho_{t}=\mathcal{N}_{\Delta}\left(\varrho_{0}\right)=\sum_{n, m} e^{-\Delta^{2}(n-m)^{2}} \varrho_{n, m}|n\rangle\langle m|,
$$

where $\Delta \equiv \Gamma t$, and $\varrho_{n, m}=\left\langle n\left|\varrho_{0}\right| m\right\rangle$. The diagonal elements are left unchanged, in fact energy is conserved, whereas the off-diagonal ones are progressively destroyed, together with the phase information carried by the state. Phase diffusion corresponds to the application of a random, zero-mean Gaussian-distributed phase shift, i.e.,

$$
\varrho_{t}=\int_{\mathbb{R}} d \beta g(\beta \mid \Delta) U_{\beta} \varrho_{0} U_{\beta}^{\dagger}, \quad g(\beta \mid \Delta)=\frac{e^{-\beta^{2} /\left(4 \Delta^{2}\right)}}{\sqrt{4 \pi \Delta^{2}}},
$$

where $U_{\beta}=\exp \left\{-i \beta\left(a^{\dagger} a\right)\right\}$ is the phase-shift operator.

We assume that the phase noise occurs between the application of the phase shift and the detection of the signal, and consider the estimation of a phase shift applied to a singlemode coherent state. Homodyne detection is then performed on the output state

$$
\varrho_{\Delta, \alpha}(\phi)=\mathcal{N}_{\Delta}\left(U_{\phi}|\alpha\rangle\langle\alpha| U_{\phi}^{\dagger}\right),
$$


and the value of the unknown phase shift $\phi$ is inferred using Bayesian estimation applied to homodyne data. Notice, however, that since the phase noise map and the phase-shift operation commute, our results are valid also when the phase shift is applied to an already phase-diffused coherent state. The precision of the above procedure is then compared with the benchmarks given by (i) the quantum CR bound for coherent states and any quantum limited kind of measurement, and (ii) the ultimate precision achievable with optimized Gaussian states, i.e., the quantum CR bound for general Gaussian signals, where, e.g., we allow for squeezing.

\section{A. Interferometric precision in the presence of phase noise}

The quantum CR bound [44-48] is obtained starting from the Born rule $p(x \mid \phi)=\operatorname{Tr}\left[\Pi_{x} \varrho_{\phi}\right]$, where $\left\{\Pi_{x}\right\}$ is the operator-valued measure describing the measurement, and $\varrho_{\phi}$ the density operator of the family of phase-shifted states under investigation. Upon introducing the (symmetric) logarithmic derivative $L_{\phi}$ as the operator satisfying

$$
2 \partial_{\phi} \varrho_{\phi}=L_{\phi} \varrho_{\phi}+\varrho_{\phi} L_{\phi},
$$

one proves that the ultimate limit to precision (independently on the measurement used) is given by the quantum CR bound

$$
\operatorname{Var}(\phi) \geqslant[M H(\phi)]^{-1},
$$

where $H(\phi)=\operatorname{Tr}\left[\varrho_{\phi} L_{\phi}^{2}\right]$ is the quantum Fisher information (QFI). The ultimate sensitivity of an interferometer thus depends on the family of signals used to probe the phase shift, and thus, as said above, we are going to compare the precision of our interferometer with the maximum achievable with coherent states, and with the ultimate precision achievable with optimized Gaussian states (for more details about the derivation of the corresponding quantum CR bounds, see [42]).

Homodyne detection measures the field quadrature

$$
x_{\theta}=\frac{1}{2}\left(a e^{-i \theta}+a^{\dagger} e^{i \theta}\right),
$$

where $\theta=\arg \alpha+\pi / 2$ is set to the optimal value to detect the imposed phase shift. The likelihood of a set of homodyne data,

$$
X=\left\{x_{1}, x_{2}, \ldots, x_{M}\right\},
$$

is the overall probability of the sample given the unknown phase $\phi$, i.e.,

$$
L(X \mid \phi)=\prod_{k=1}^{M} p\left(x_{k} \mid \phi\right)
$$

where

$$
p(x \mid \phi)=\frac{e^{-2 x^{2}}}{\pi \Delta} \int_{\mathbb{R}} d \beta e^{-\frac{\beta^{2}}{2 \Delta^{2}}+4 \alpha x \cos (\beta+\phi)-2 \alpha^{2} \cos ^{2}(\beta+\phi)} .
$$

Assuming that no a priori information is available on the value of the phase shift (i.e., uniform prior), and using the Bayes theorem, one can write the a posteriori probability

$$
P(\phi \mid X)=\frac{1}{\mathcal{N}} L(X \mid \phi), \quad \mathcal{N}=\int_{\Phi} d \phi L(\phi \mid X),
$$

$\Phi=[0, \pi]$ being the parameter space. The probability $P(\phi \mid X)$ is the expected distribution of $\phi$ given the data sample $X$.
The Bayesian estimator $\phi_{\mathrm{B}}$ is the mean of the a posteriori distribution, whereas the sensitivity of the overall procedure corresponds to its variance

$$
\operatorname{Var}\left[\phi_{\mathrm{B}}\right]=\int_{\Phi} d \phi\left(\phi-\phi_{\mathrm{B}}\right)^{2} P(\phi \mid X) .
$$

Bayesian estimators are known to be asymptotically unbiased and optimal, namely, they allow one to achieve the CR bound as the size of the data sample increases $[49,50]$. On the other hand, the number of data needed to achieve the asymptotic region may depend on the specific implementation [51]. In the following we will experimentally show that our setup achieves optimal estimation already after collecting a few hundreds of measurements.

\section{EXPERIMENTAL APPARATUS}

A schematic diagram of the interferometer is reported in Fig. 1. The principal radiation source is provided by a He:Ne laser (12 mW, $633 \mathrm{~nm})$ shot-noise limited above $2 \mathrm{MHz}$. The laser emits a linearly polarized beam in a TEM00 mode. The beam is split into two parts of variable relative intensity by a combination of a half wave plate (HWP) and a polarizing beam splitter (PBS). The strongest part is sent directly to the homodyne detector where it acts as the local oscillator, whereas the remaining part is used to encode the signal and will undergo the homodyne detection. The optical paths traveled by the local oscillator and the signal beams are carefully adjusted to obtain a visibility typically above $90 \%$ measured at one of the homodyne output ports. The signal is amplitude modulated at $4 \mathrm{MHz}$ with a defined modulation depth to control the average number of photons in the generated state.

The amplitude modulation system consists of a potassium dihydrogen phosphate (KDP) nonlinear crystal with the $x y$

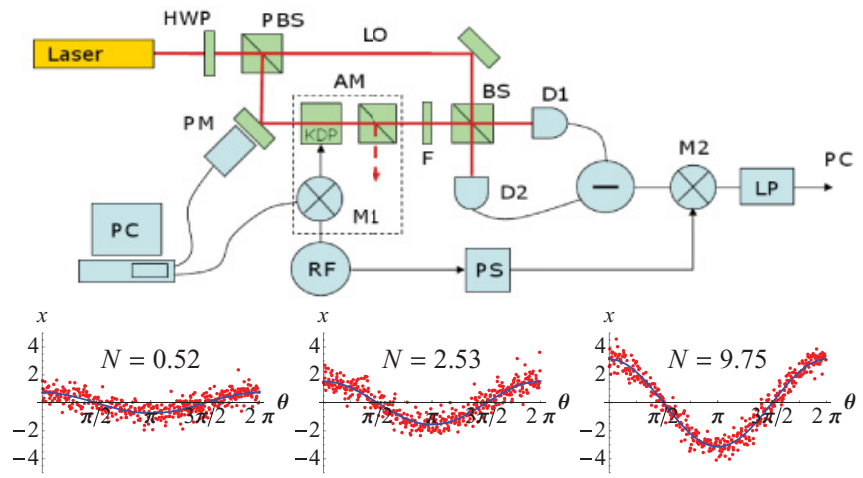

FIG. 1. (Color online) Schematic diagram of the experimental setup. A He:Ne laser is divided into two beams, one acts as the local oscillator and the other represents the signal beam. The signal is modulated at $4 \mathrm{MHz}$ with a defined modulation depth to control the average number of photons in the generated state. One of the mirrors in the signal path is piezo mounted to obtain a variable phase difference between the two beams. The data are recorded by a homodyne detector whose difference photocurrent is demodulated and then acquired by a computer after a low pass filter. We also show the typical homodyne samples obtained for coherent signals of different amplitudes by varying the phase of the local oscillator (these are used to check the calibration of the piezo, which is performed using signals with a larger number of photons). 
axes at $45^{\circ}$, and a PBS. The modulation is applied at the KDP crystal by means of a Rohde \& Schwarz wave form generator and a Mini-Circuits ZHL-32A power amplifier. The modulation depth is imposed at the proper level by a computer that sends a constant voltage to a mixer (M1) located between the wave form generator and the power amplifier. One of the mirrors in the signal path is piezo mounted to obtain a variable phase difference between the two beams. The piezo is preloaded and its resonance frequency is $13.5 \mathrm{kHz}$.

The phase difference is controlled by the computer after a calibration stage. The computer sends a voltage signal between 0 and $10 \mathrm{~V}$ that corresponds at the phase diffusion with a frequency of $5 \mathrm{kHz}$ to a power amplifier based on an LM675 integrated circuit that is able to drive the piezo at this frequency. With this system it is possible to generate any kind of phase modulation.

The detector is composed by a 50:50 beam splitter (BS) and a balanced amplifier detector with a bandwidth of $50 \mathrm{MHz}$. The difference photocurrent is filtered with high pass filters, amplified and demodulated at $4 \mathrm{MHz}$ by means of an electrical mixer (M2). In this way the detection occurs outside any technical noise and, more importantly, in a spectral region where the laser does not carry excess noise. The signal is filtered by a low pass filter with a bandwidth of $300 \mathrm{kHz}$ and sent to the computer through the National Instrument multichannel data acquisition 6251 with a 16-bit resolution and a $1.25 \mathrm{MS} / \mathrm{s}$ sampling rate. The same device is used to send diffusion parameters to the phase modulator and signal parameters to the amplitude modulator.

\section{EXPERIMENTAL RESULTS}

In this section, we present our experimental results, obtained with signals of different energies and different levels of noise. At first we show homodyne samples with the corresponding a posteriori distributions and then compare the precision obtained in our scheme with the ultimate bound imposed by the (quantum) Cramér-Rao theorem. Finally, we analyze the dependence of precision on the signal energy and the noise in order to illustrate how in the limit of large phase diffusion, coherent states becomes the optimal Gaussian probe states. In fact, they outperform squeezed vacuum states, whose nonclassical features are degraded by the phase diffusion process, to an extent that make them useless for quantum metrology.

In Fig. 2 we report typical examples of homodyne samples, referred to a coherent signal with $N=|\alpha|^{2}$ mean photon number measured at fixed optimal $\theta$, together with the corresponding Bayesian a posteriori distribution for the phase shift. The yellow areas denote the portions of data used to infer the phase shifts. We choose this range in order to emphasize that the optimality region is achieved already in that region. In fact, upon considering larger samples, precision would be improved, due to the statistical scaling of the variance $\operatorname{Var}[\phi]=C / M, C$ being a proportionality constant. On the other hand, optimality, i.e., the fact that

$$
C \simeq 1 / H_{\alpha},
$$

where $H_{\alpha}$ is the QFI for phase-diffused coherent signals, is achieved for $M \sim 100$ measurements. In the noiseless case the
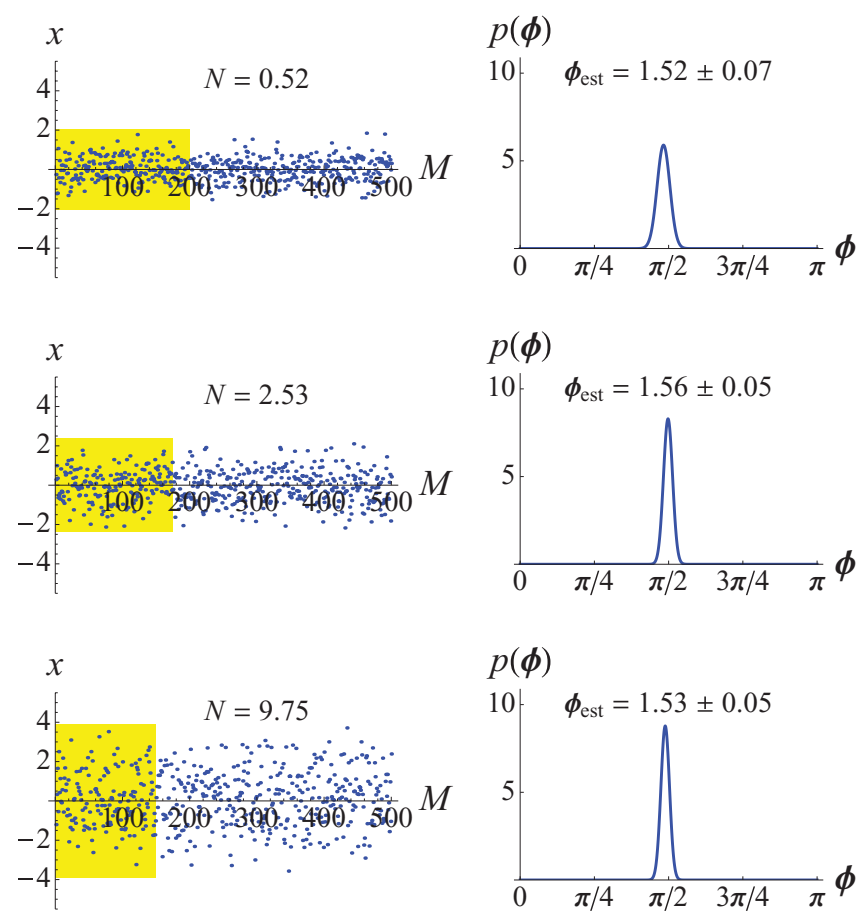

FIG. 2. (Color online) Typical examples of homodyne samples measured at fixed optimal $\theta$, together with the corresponding Bayesian a posteriori distribution for the phase shift. The phase diffusion is $\Delta=\pi / 6 \mathrm{rad}$, and the yellow areas denote the portions of the data used to infer the phase shifts.

QFI is given by $H_{\alpha}=4 N$, whereas it decreases monotonically by increasing the value of the noise parameter $\Delta$. Notice that using optimized Gaussian signals, i.e., the squeezed vacuum state, one has a QFI given by $H_{g}=8 N^{2}+8 N$ in the noiseless case. However, in the presence of large phase diffusion, i.e., for large values of $\Delta, H_{\alpha}$ is larger than the QFI obtained for phasediffused squeezed vacuum states. In other words, coherent states turn out to be the optimal Gaussian probe states [42].

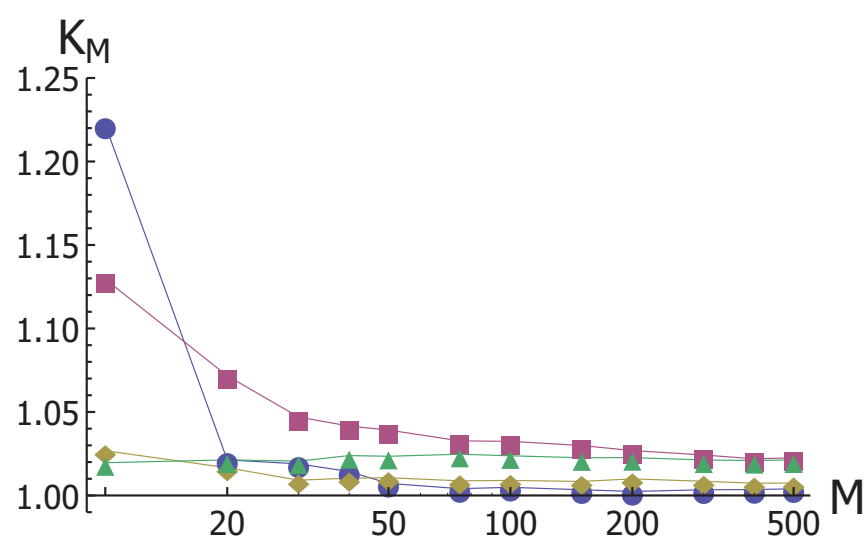

FIG. 3. (Color online) The noise ratio $K_{M}=\left(\operatorname{Var}\left[\phi_{\mathrm{B}}\right] M H_{\alpha}\right)$ as a function of the number of data $M$ and for different values of the number of photons $N$ and the noise parameter $\Delta$. Blue circles: $N=$ $0.90, \Delta=\pi / 18 \mathrm{rad}$; red squares: $N=0.90, \Delta=\pi / 9 \mathrm{rad}$; yellow diamonds: $N=4.12, \Delta=\pi / 18 \mathrm{rad}$; green triangles: $N=4.12, \Delta=$ $\pi / 9 \mathrm{rad}$. 
In Fig. 3 we plot the quantity

$$
K_{M}=M \operatorname{Var}\left[\phi_{\mathrm{B}}\right] H_{\alpha},
$$

i.e., the variance of the Bayesian estimator from homodyne data multiplied by the number of data (measurements) and by the coherent states quantum Fisher information, as a function of $M . K_{M}$ is by definition larger than 1 and expresses the ratio between the actual precision of the interferometric setup and the CR bound. As it is apparent from the plot $K_{M}$ rapidly decreases with the number of measurements, almost independently of the value of the number of photons $N$ and of the noise parameter $\Delta$. The optimality region, i.e., $K_{M} \simeq 1$ is achieved already for $M \simeq 100$ measurements, and the asymptotic value of $K_{M}$ is closer to 1 for increasing $N$
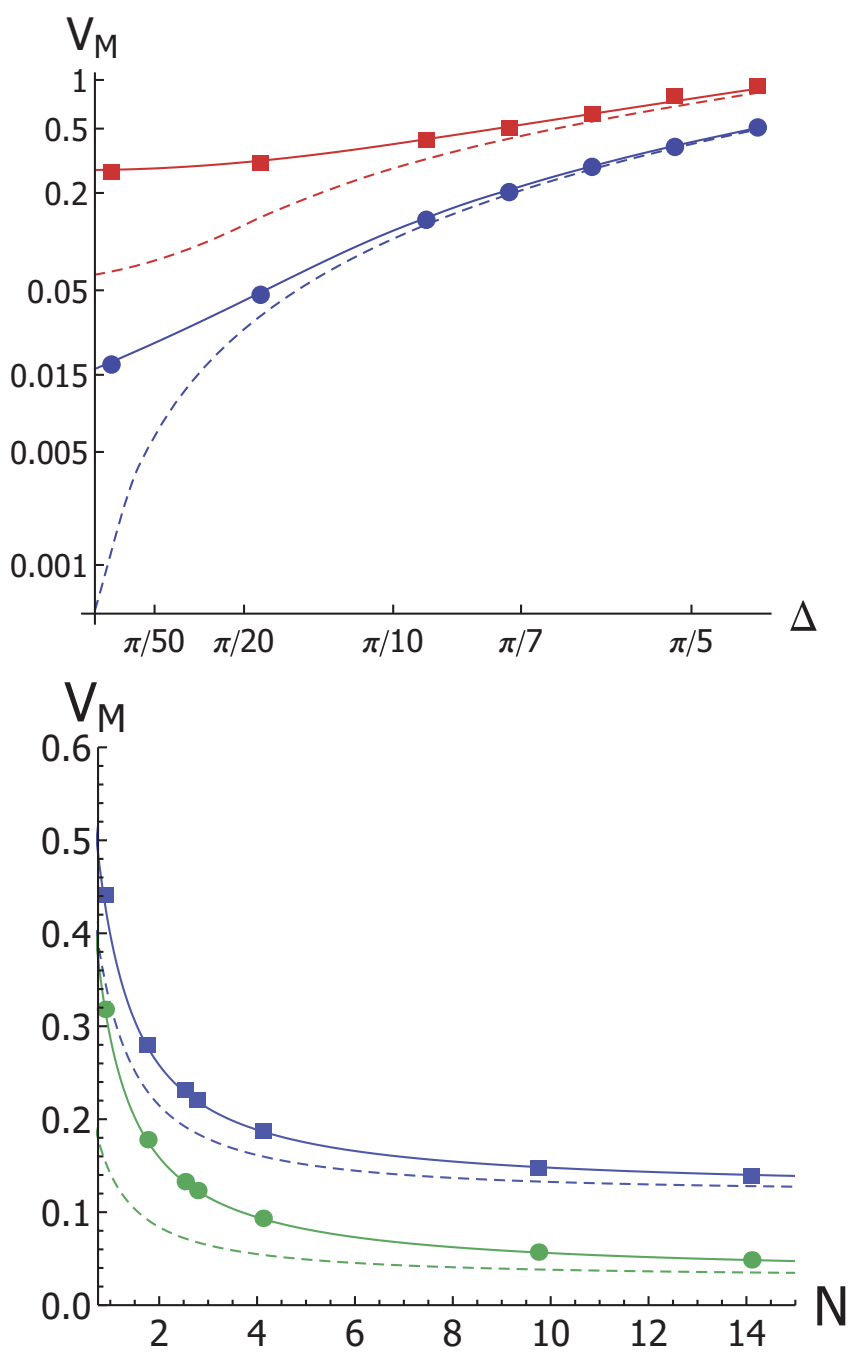

FIG. 4. (Color online) Variance $V_{M}=M \operatorname{Var}\left[\phi_{\mathrm{B}}\right]$ of the Bayesian estimator from homodyne data after $M=100$ measurements (points), together with the CR bound for coherent states (solid lines) and for optimized Gaussian states (dashed lines). The top panel shows the behavior of $V_{100}$ as a function of $\Delta$ for different values of the number of photons (top red lines/squares: $N=0.90$; bottom blue lines/circles: $N=14.11$ ). The bottom panel shows $V_{100}$ as a function of the number of photons $N$ and for different values of the noise (top blue lines/squares: $\Delta=\pi / 9 \mathrm{rad}$; bottom green lines/circles: $\Delta=\pi / 18 \mathrm{rad})$. and $\Delta$. Furthermore, the number of measurements needed to achieve the optimal region may be (slightly) reduced by using the Jeffreys prior [52]

$$
p(\phi) \propto \sqrt{F(\phi)}
$$

instead of the uniform one, where

$$
F(\phi)=\int d x p(x \mid \phi)\left[\partial_{\phi} \ln p(x \mid \phi)\right]^{2}
$$

is the Fisher information of the homodyne distribution.

In Fig. 4 we show the variance of the Bayesian estimator from homodyne data,

$$
V_{M}=M \operatorname{Var}\left[\phi_{\mathrm{B}}\right],
$$

obtained after $M$ measurements, together with the CR bound $1 / H_{\alpha}$ for coherent states, and for the (phase-diffused) optimized Gaussian states, i.e., $1 / H_{g}$. In particular, the top panel shows the behavior as a function of $\Delta$ for different values of the number of photons $N$, while in the bottom panel we plot the same quantities as a function of the number of photons $N$ and for different values of the noise $\Delta$. As it is apparent from the plots, nearly optimal interferometric precision is achieved for increasing energy or phase diffusion, i.e., for larger values of $N$ or $\Delta$.

\section{CONCLUSIONS}

In conclusion, we have demonstrated a nearly optimal interferometric scheme based on homodyne detection and coherent signals for the detection of a phase shift in the presence of large phase diffusion. Our scheme does not require nonclassical light and achieves the ultimate bound to interferometric sensitivity using Bayesian analysis on small samples of homodyne data, where the number of measurements is of the order of a few hundreds.

It is worth noting that for large phase diffusion, coherent states are the optimal Gaussian probe states. Indeed they outperform squeezed vacuum states, whose nonclassical features are degraded by the phase diffusion process, such that they become completely useless for quantum metrology.

Optical interferometry represents a highly accurate measurement scheme with wide applications in many fields of science and technology, including high-precision measurements and communication channels. On the other hand, phase diffusion represents a crucial obstacle toward the implementation of high-precision interferometric measurements and phase-shiftbased communication channels. Our results allow one to design feasible, high-performance, communication channels also in the presence of phase noise, which cannot be effectively controlled in realistic conditions. Therefore, besides fundamental interest, our results also represent a benchmark for realistic phase based communication or measurement protocols.

\section{ACKNOWLEDGMENTS}

This work has been supported by MIUR (FIRB "LiCHIS" Grant No. RBFR10YQ3H), the UK EPSRC (EP/I026436/1), MAE (INQUEST), UniMi (PUR2009 SIN.PHO.NANO), UIF/UFI (Vinci Program), and the University of Trieste (FRA2009). 
[1] C. M. Caves, Phys. Rev. D 23, 1693 (1981).

[2] R. Bluhm, V. A. Kostelecky, C. D. Lane, and N. Russell, Phys. Rev. Lett. 88, 090801 (2002).

[3] C. Jentsch, T. Muller, E. Rasel, and W. Ertmer, Gen. Relativ. Gravit. 36, 2197 (2004).

[4] S. Fray, C. A. Diez, T. W. Hänsch, and M. Weitz, Phys. Rev. Lett. 93, 240404 (2004).

[5] M. J. Snadden, J. M. McGuirk, P. Bouyer, K. G. Haritos, and M. A. Kasevich, Phys. Rev. Lett. 81, 971 (1998).

[6] M. A. Armen, J. K. Au, J. K. Stockton, A. C. Doherty, and H. Mabuchi, Phys. Rev. Lett. 89, 133602 (2002).

[7] M. W. Mitchell, J. S. Lundeen, and A. M. Steinberg, Nature (London) 429, 161 (2004).

[8] T. Nagata, R. Okamoto, J. L. O’Brien, K. Sasaki, and S. Takeuchi, Science 316, 726 (2007).

[9] K. J. Resch, K. L. Pregnell, R. Prevedel, A. Gilchrist, G. J. Pryde, J. L. O’Brien, and A. G. White, Phys. Rev. Lett. 98, 223601 (2007).

[10] B. L. Higgins, D. W. Berry, S. D. Bartlett, H. M. Wiseman, and G. J. Pryde, Nature (London) 450, 393 (2007).

[11] B. L. Higgins, D. W. Berry, S. D. Bartlett, M. W. Mitchell, H. M. Wiseman, and G. J. Pryde, New J. Phys. 11, 073023 (2009).

[12] Z. Hradil, Quantum Opt. 4, 93 (1992).

[13] S. L. Braunstein, Phys. Rev. Lett. 69, 3598 (1992).

[14] A. S. Lane, S. L. Braunstein, and C. M. Caves, Phys. Rev. A 47, 1667 (1993).

[15] B. C. Sanders and G. J. Milburn, Phys. Rev. Lett. 75, 2944 (1995).

[16] K. Eckert, P. Hyllus, D. Bruss, U. V. Poulsen, M. Lewenstein, C. Jentsch, T. Muller, E. M. Rasel, and W. Ertmer, Phys. Rev. A 73, 013814 (2006).

[17] V. Giovannetti, S. Lloyd, and L. Maccone, Science 306, 1330 (2004); Phys. Rev. Lett. 96, 010401 (2006).

[18] F. W. Sun, B. H. Liu, Y. X. Gong, Y. F. Huang, Z. Y. Ou, and G. C. Guo, Europhys. Lett. 82, 24001 (2008).

[19] L. Pezzé, A. Smerzi, G. Khoury, J. F. Hodelin, and D. Bouwmeester, Phys. Rev. Lett. 99, 223602 (2007); L. Pezzé and A. Smerzi, ibid. 100, 073601 (2008).

[20] J. Grond, U. Hohenester, I. Mazets, and J. Schmiedmyer, New J. Phys. 12, 065036 (2010); J. Grond, U. Hohenester, J. Schmiedmayer, and A. Smerzi, Phys. Rev. A 84, 023619 (2011).

[21] M. Hayashi, Progr. Inf. 8, 81 (2011).

[22] D. Braun and J. Martin, Nat. Comm. 2, 223 (2011); D. Braun, Eur. Phys. J. D 59, 521 (2010).

[23] S. Boixo, A. Datta, M. J. Davis, S. T. Flammia, A. Shaji, and C. M. Caves, Phys. Rev. Lett. 101, 040403 (2008).

[24] V. Giovannetti, S. Lloyd, and L. Maccone, Nat. Phot. 5, 222 (2011).

[25] M. G. A. Paris, Phys. Lett. A 201, 132 (1995); S. Olivares and M. G. A. Paris, Optics Spectr. 103, 231 (2007).

[26] R. A. Campos, C. C. Gerry, and A. Benmoussa, Phys. Rev. A 68, 023810 (2003).
[27] S. D. Huver, C. F. Wildfeuer, and J. P. Dowling, Phys. Rev. A 78, 063828 (2008).

[28] J. J. Cooper, D. W. Hallwood, and J. A. Dunningham, Phys. Rev. A 81, 043624 (2010).

[29] M. Kacprowicz, R. Demkowicz-Dobrzanski, W. Wasilewski, K. Banaszek, and I. A. Walmsley, Nat. Phot. 4, 357 (2010).

[30] U. Dorner, R. Demkowicz-Dobrzanski, B. J. Smith, J. S. Lundeen, W. Wasilewski, K. Banaszek, and I. A. Walmsley, Phys. Rev. Lett. 102, 040403 (2009); R. Demkowicz-Dobrzanski, U. Dorner, B. J. Smith, J. S. Lundeen, W. Wasilewski, K. Banaszek, and I. A. Walmsley, Phys. Rev. A 80, 013825 (2009).

[31] H. Cable and G. A. Durkin, Phys. Rev. Lett. 105, 013603 (2010).

[32] J. Joo, W. J. Munro, and T. P. Spiller, Phys. Rev. Lett. 107, 083601 (2011).

[33] S. Knysh, V. N. Smelyanskiy, and G. A. Durkin, Phys. Rev. A 83, 021804(R) (2011).

[34] T. B. Bahder, Phys. Rev. A 83, 053601 (2011).

[35] A. Datta, L. Zhang, N. Thomas-Peter, U. Dorner, B. J. Smith, and I. A. Walmsley, Phys. Rev. A 83, 063836 (2011).

[36] D. Brivio, S. Cialdi, S. Vezzoli, B. T. Gebrehiwot, M. G. Genoni, S. Olivares, and M. G. A. Paris, Phys. Rev. A 81, 012305 (2010).

[37] B. Teklu, M. G. Genoni, S. Olivares, and M. G. A. Paris, Phys. Scr. T 140, 014062 (2010).

[38] E. Tesio, S. Olivares, and M. G. A. Paris, Int. J. Quantum. Inform. 9, 379 (2011).

[39] I. Tikhonenkov, M. G. Moore, and A. Vardi, Phys. Rev. A 82, 043624 (2010).

[40] Y. C. Liu, G. R. Jin, and L. You, Phys. Rev. A 82, 045601 (2010).

[41] G. Ferrini, D. Spehner, A. Minguzzi, and F. W. J. Hekking, Phys. Rev. A 82, 033621 (2010).

[42] M. G. Genoni, S. Olivares, and M. G. A. Paris, Phys. Rev. Lett. 106, 153603 (2011).

[43] A. Monras, Phys. Rev. A 73, 033821 (2006).

[44] J. D. Malley and J. Hornstein, Stat. Sci. 8, 433 (1993).

[45] S. L. Braunstein and C. M. Caves, Phys. Rev. Lett. 72, 3439 (1994); S. L. Braunstein, C. M. Caves, and G. J. Milburn, Ann. Phys. (NY) 247, 135 (1996).

[46] D. C. Brody and L. P. Hughston, Proc. R. Soc. London A 454, 2445 (1998); 455, 1683 (1999).

[47] M. G. A. Paris, Int. J. Quantum. Inform. 7, 125 (2009).

[48] B. M. Escher, R. L. de Matos Filho, and L. Davidovich, Nat. Phys. 7, 406 (2011); B. M. Escher, R. L. de Matos Filho, and L. Davidovich, Braz. J. Phys. 41, 229 (2011).

[49] Z. Hradil, Phys. Rev. A 51, 1870 (1995); Z. Hradil, R. Myška, J. Peřina, M. Zawisky, Y. Hasegawa, and H. Rauch, Phys. Rev. Lett. 76, 4295 (1996).

[50] S. Olivares and M. G. A. Paris, J. Phys. B 42, 055506 (2009); B. Teklu, S. Olivares, and M. G. A. Paris, ibid. 42, 035502 (2009).

[51] O. E. Barndorff-Nielsen and R. D. Gill, J. Phys. A 33, 4481 (2000).

[52] H. Jeffreys, Proc. R. Soc. London A186, 453 (1946). 